HIFAN 1660

\title{
Testing of optical diagnostics for ion-beam-driven WDM experiments at NDCX-1
}

\author{
P. A. Ni, F. M. Bieniosek, M. Leitner, C. Weber, and W. L. Waldron \\ LBNL
}

Accelerator Fusion Research Division

Ernest Orlando Lawrence Berkeley National Laboratory

University of California

Berkeley, California 94720

June 2007

This work was supported by the Director, Office of Science, Office of Fusion Energy Sciences, of the U.S.

Department of Energy under Contract No. DE-AC02-05CH11231. 


\title{
Testing of optical diagnostics for ion-beam-driven WDM experiments at NDCX-1
}

\author{
P. A. Ni, ${ }^{1}$ F. M. Bieniosek, ${ }^{1}$ M. Leitner, ${ }^{1}$ C. Weber,${ }^{1}$ and W. L. Waldron ${ }^{1}$ \\ ${ }^{1}$ Lawrence Berkeley National Laboratory, University of California, Berkeley, California, USA
}

(Dated: August 20, 2008)

\begin{abstract}
We report on the testing of optical diagnostics developed for warm-dense-matter (WDM) experiments on the Neutralized Drift Compression Experiments (NDCX-1) at Lawrence Berkeley National Laboratory (LBNL). The diagnostics consist of a fast optical pyrometer, a streak camera spectrometer, and a VISAR. While the NDCX is in the last stage of commissioning for the target experiments, the diagnostics were tested elsewhere in an experiment where an intense laser pulse was used to generate the WDM state in metallic and carbon samples.
\end{abstract}

PACS numbers:

\section{INTRODUCTION}

The planned warm-dense-matter experiments at NDCX-1 [1, 2] require sub-nanosecond optical diagnostics which are capable of measuring brightness temperature from $0.1 \mathrm{eV}$ to $1 \mathrm{eV}$ in the visible and near-infrared parts of spectrum and expansion velocity up to several $\mathrm{km} / \mathrm{s}$. The requirement for probing spatial resolution is dictated by the ion beam spot size $(\approx 1 \mathrm{~mm})$ and must be on the order of several of several hundred of micrometers.

We have developed the first prototypes of diagnostics, which consists of an fast optical pyrometer, streak camera spectrometer and a Doppler-shift laser interferometer (VISAR).

During the final tuning of the NDCX-1 beam [3, 4], we tested the equipment in laser heating experiments at the beam line 6.0.2 of Advanced Light Source at LBNL.

\section{EXPERIMENTAL SETUP}

We used an amplified Ti:Sapphire laser at $\lambda=800 \mathrm{~nm}$ with 150 fs pulse duration, pulse energy tuned in the range of 1-5 mJ, spot size $400-500 \mu \mathrm{m}$, and angle of incidence 30 degrees. Targets were free-standing foils of amorphous carbon (50 nm thick) or copper (120 nm thick, with $5 \mathrm{~nm}$ of carbon on each side to prevent oxidation) on a supporting Ni mesh with $360 \mu \mathrm{m}$ spacing.

The probing optics consisted of two coated, fast, achromatic, VIS-NIR doublets $(\mathrm{d}=50.8 \mathrm{~mm}, \mathrm{f}=75 \mathrm{~mm})$, which collected thermal light from a $200 \mu \mathrm{m}$ spot at the target and coupled it to a multimode optical fiber $(\mathrm{d}=200 \mu \mathrm{m})$ that was connected either to the pyrometer or the streak camera spectrometer.

\section{FAST OPTICAL PYROMETER}

A specially developed three-channel pyrometer probes color temperatures at $750 \mathrm{~nm}, 1000 \mathrm{~nm}$ and $1500 \mathrm{~nm}$. The pyrometer design is based on custom spectrally selective beam splitters and can be upgraded up to seven channels. Each beam splitter reflects a spectral band 150

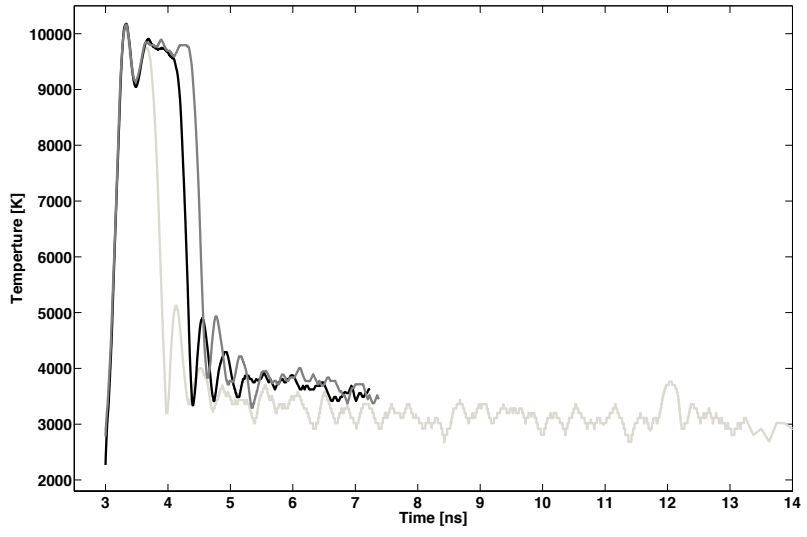

FIG. 1: Brightness temperature at $1000 \mathrm{~nm}$ recorded for three nominally identical shots (represented by different colors), with a $200 \mathrm{~nm}$ thick copper target.

nm wide and transmits the rest. The spectrally discriminated light is then coupled to an amplified photo-receiver. A Si PIN diode is used as a detector for the visible part of spectrum and an InGaAs PIN diode for the near-infrared part. The distinctive feature of the detectors is that they have a flat gain curve from DC to $4 \mathrm{GHz}$ with 75 ps rise time.

The pyrometer is absolutely calibrated with a tungsten ribbon lamp (NIST traceable) at $2600 \mathrm{~K}$. An average voltage response of the pyrometer at this temperature is $2 \mathrm{mV}$, with $0.2 \mathrm{mV}$ rms of the noise. The estimated detection bounds are 2000-100000 K (black body).

The temperature records of an experiment where the Ti-Sapphire laser heated a copper foil are shown in figures 1 and 2 . Figure 1 demonstrates a temperature evolution during the first $15 \mathrm{~ns}$ while figure 2 shows the thermal radiation recorded on a longer $300 \mathrm{~ns}$ time scale.

The laser pulse duration is 150 fs and the hydrodynamic response time is on the 50 ps time scale. Given the numbers, one would generally expect a rapid monotonic decrease in temperature due to expansion. However a temperature plateau around $10000 \mathrm{~K}$ lasting for $2 \mathrm{~ns}$ and followed by a rapid temperature drop can be observed in 


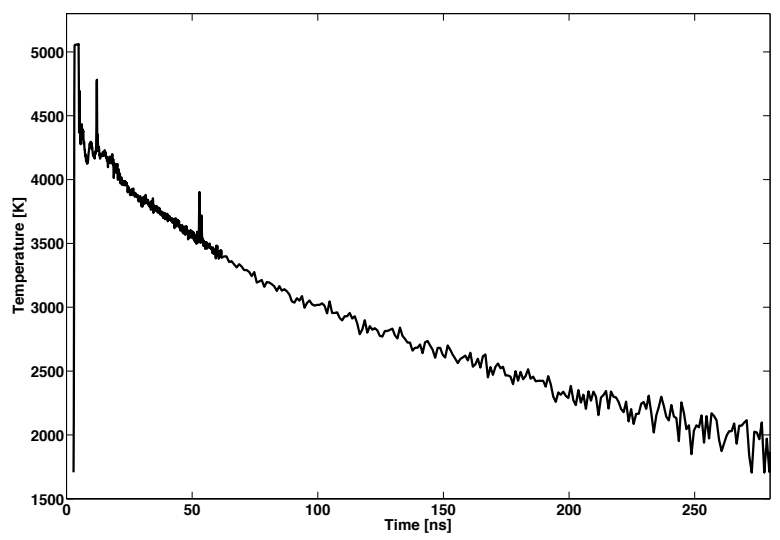

FIG. 2: Brightness temperature at $1000 \mathrm{~nm}$ of a laser-heated, $200 \mathrm{~nm}$ thick, copper foil (long record). Unlike the response at short times, there is no significant shot-to-shot variation.

figure 1. In order to understand this phenomenon, hydrodynamic simulations of the performed experiments with the DISH code, which includes a radiation transport in WDM, are planned [5].

\section{STREAK CAMERA SPECTROMETER}

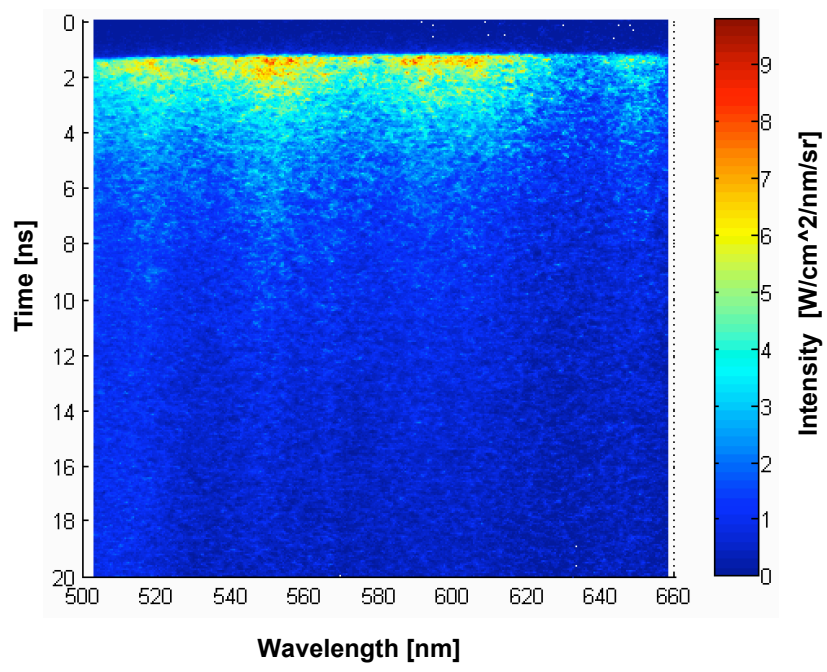

FIG. 3: Absolute radiation spectrum of a laser heated carbon foil recorded by a streak camera spectrometer.

Continuous target emission from $450 \mathrm{~nm}$ to $850 \mathrm{~nm}$ was recorded by a custom spectrometer, consisting of a highdynamic-range Hamamatsu C7700 streak camera and a holographic fiber-coupled grating etched on an off-axis parabolic mirror. Similarly to the pyrometer, the spectrometer is calibrated absolutely with the tungsten rib- bon lamp. The spectrally resolved radiation of a laser heated carbon foil is shown in figure 3 .

\section{VISAR}

We have also performed an off-line test of the commercially available all-fiber Doppler shift laser interferometer. The purpose of the VISAR is to measure the expansion velocity of a target surface, which is related to pressure of a target and therefore can lead to pressure determination.

The foils of $\mathrm{Cu}$ and amorphous carbon used in the pyrometry measurements had many small wrinkles. So to provide a reflective and fast moving surface for the test, we used a modified nail gun typically used for construction work. A polished aluminum foil was glued to the air propelled piston of the gun and the probing laser beam was focused and aligned normally to the foil. The reflected beam was fed back into the interferometer using the same focusing optics. VISAR records obtained during the test are shown in figure 4 .

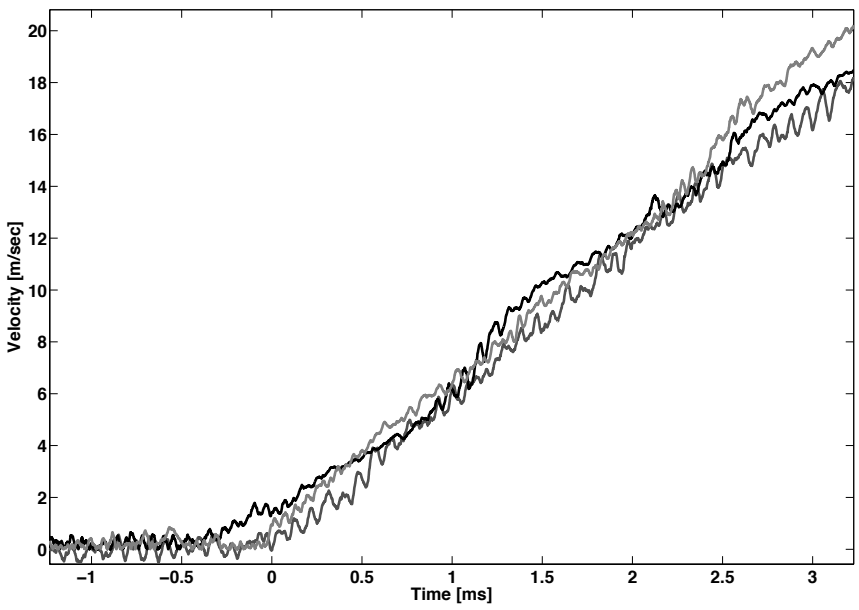

FIG. 4: Piston velocity of a nail gun measured by VISAR for three different shots (different colors represent different shots).

\section{SUMMARY}

We have designed the first working prototypes of planned optical diagnostics and tested them in laser heated target experiments. The operational experience gained is of a great value for the planed WDM experiments on NDCX-1. Further improvements in electromagnetical shielding, sensitivity, resolution and speed are planned. 
[1] F.M. Bieniosek, et. al. Nucl. Instrum. Methods Phys. Res. A544 (2005) 268-276.

[2] F. M. Bieniosek, J. J. Barnard, J.E. Coleman, E. Henestroza, M. A. Leitner,, B. G. Logan, R. M. More, P. A. Ni, P. K. Roy, W. L. Waldron, P. A. Seidl, "High energy density physics experiments with intense heavy ion beams". Proceedings to this conference.

[3] P. A. Seidl, A. Anders, R.H. Cohen, J.E. Coleman,, M. Dorf, E.P. Gilson, D.P. Grote, J.Y. Jung, M. Leitner, S.M. Lidia, B.G. Logan, P.K. Roy, A.B. Sefkow, W.L. Waldron, D.R. Welch, "Progress in beam focusing and compression for warm dense matter experiments" Proceedings to this conference.
[4] P. K. Roy, P. A. Seidl, A. Anders, J. E. Coleman,, E. P. Gilson, D. P. Grote, J. Y. Jung, M. Leitner, S. Lidia, B. G. Logan, A. B. Sefkow, W. L. Waldron, D. R. Welch , "Space-charge neutralizing plasma for beam drift compression experiments", Proceedings to this conference.

[5] J. J. Barnard, J. Armijo, D. S. Bailey, A. Friedman, F. M. Bieniosek, E. Henestroza, I. Kaganovich, P. T. Leung, B. G. Logan, M.M. Marinak, R. M. More, S. F. Ng,, G. E. Penn, L. J. Perkins, P. Stoltz, S. Veitzer, J. S. Wurtele, S. S. Yu, A. B. Zylstra "Ion beam heated target simulations and analysis for warm dense matter physics and inertial fusion energy". Proceedings to this conference. 